OPEN ACCESS

Edited by:

Aiping Liu,

The University of British Columbia,

Canada

Reviewed by:

Yi Li,

New York University, United States

Niels Janssen,

Universidad de La Laguna, Spain

${ }^{*}$ Correspondence:

Bin-Yin L

libinyin@126.com

Sheng-Di Chen

chensd@rih.com.cn

Received: 06 September 2018

Accepted: 21 January 2019

Published: 05 February 2019

Citation:

Lin Y-Q, Cui S-S, Du J-J, Li G He $Y$-X, Zhang $P-C$, Fu Y, Huang $P$, Gao C, Li B-Y and Chen S-D (2019) $\mathrm{N} 1$ and $\mathrm{P} 1$ Components Associate

With Visuospatial-Executive and Language Functions in Normosmic Parkinson's Disease: An Event-Related Potential Study.

Front. Aging Neurosci. 11:18.

doi: 10.3389/fnagi.2019.00018

\section{N1 and P1 Components Associate With Visuospatial-Executive and Language Functions in Normosmic Parkinson's Disease: An Event-Related Potential Study}

\author{
Yi-Qi Lin, Shi-Shuang Cui, Juan-Juan Du, Gen Li, Yi-Xi He, Ping-Chen Zhang, Yang Fu, \\ Pei Huang, Chao Gao, Bin-Yin Li* and Sheng-Di Chen*
}

Department of Neurology and Institute of Neurology, Ruijin Hospital Affiliated to Shanghai Jiao Tong University School of Medicine, Shanghai, China

Background: Hyposmia is one of the most important clinical markers of Parkinson's disease (PD) with a prevalence ranging from 50 to $96 \%$ of PD patients. A significant association was found between hyposmia and cognitive impairment of PD. However, there were no reports of event-related potentials (ERP) performance in PD patients with and without hyposmia for cognitive functions assessment.

Purpose: The aim of our study was to compare ERP performance and its association with cognitive domains between PD with and without hyposmia.

Methods: Olfactory functions were assessed by Sniffin' Sticks test-16 (SS-16). Twentyfour subjects were included in PD with hyposmia group and nineteen were in PD without hyposmia group. ERP measures were recorded during a delayed match to sample (DMS) task with Chinese characters. The parameters of ERP components including $\mathrm{N} 1, \mathrm{~N} 2, \mathrm{P} 1, \mathrm{P} 2$, and $\mathrm{P} 3$ in retrieval epoch were compared between the two groups and the correlation between ERP results and MOCA item score was also analyzed.

Results: No significant difference was found in ERP performance between PD with and without hyposmia. Among all participants, N1 latency was significantly negatively related to visuospatial-executive item score of Montreal Cognitive Assessment (MOCA) $\left(r_{\mathrm{S}}=-0.381, P=0.012\right)$ and $\mathrm{P} 1$ amplitude was positively associated with language item score of MOCA $\left(r_{\mathrm{S}}=0.302, P=0.049\right)$. Within the normosmic group, a significant association was found between $\mathrm{N} 1$ latency and visuospatial-executive item score $\left(r_{\mathrm{s}}=-0.619, P=0.005\right)$ and there was also a correlation between language score and P1 amplitude $\left(r_{s}=0.537, P=0.018\right)$. In the hyposmic group, only a significant correlation was found between $\mathrm{N} 1$ latency and clock drawing test performance $\left(r_{\mathrm{s}}=-0.413\right.$, $P=0.045)$ rather than visuospatial-executive item score. Furthermore, SS-16 score was not found to be significantly associated with either visuospatial-executive or language item score of MOCA. 


\begin{abstract}
Conclusion: No significant difference was found in ERP components between PD with and without hyposmia. N1 latency and P1 amplitude were respectively associated with visuospatial-executive and language functions in the normosmic group while in the hyposmic group, only a significant correlation was found between N1 latency and clock drawing test performance rather than visuospatial-executive item score in MOCA.
\end{abstract}

Keywords: hyposmia, Parkinson's disease, event-related potentials, working memory, visuospatial function, language

\section{INTRODUCTION}

Hyposmia is one of the most important non-motor symptoms of Parkinson's disease (PD) with a prevalence ranging from 50 to $96 \%$ of PD patients (Doty et al., 1988; Wenning et al., 1995; Hawkes et al., 1997; Boesveldt et al., 2008; Duda, 2010). It generally predates motor symptoms and the olfactory testing is useful in differentiating PD from non-PD patients with a sensitivity ranging from 79 to $100 \%$ and a specificity from 80 to $89 \%$ (Doty, 2012). Therefore, hyposmia is reported to be a critical clinical marker of PD.

In recent years olfactory dysfunction is found to be associated with other non-motor symptoms in PD patients such as chronic constipation, clinical possible rapid eye movement behavior disorder (RBD) (Chen et al., 2015) and psychotic symptoms (Morley et al., 2011). Notably, increasing evidence suggests that hyposmia is strongly associated with cognitive impairment in $\mathrm{PD}$ patients and may be a risk factor for PD dementia (Bohnen et al., 2010; Stephenson et al., 2010; Morley et al., 2011; Parrao et al., 2012; Domellof et al., 2017). In fact, the association between olfaction and cognition has been long reported in mild cognitive impairment and Alzheimer's disease which was evidenced by prominent atrophy in the primary olfactory cortex and hippocampus (Vasavada et al., 2015) as well as a strong correlation between tau pathology in the olfactory bulb and limbic systems (Attems et al., 2005). However, the role of hyposmia in cognitive impairment in $\mathrm{PD}$ patients is not clear.

Event-related potentials (ERP) have been widely used for assessing cognitive functions and brain ability. ERP wave latency and amplitude represent respectively the length of time spent and the amount of neural resources participating during information processing. There were increasing studies of ERP in PD patients owning to its independence of motor speed and disability. The abnormal P300 was reported to be associated with cognitive impairment in PD (Katsarou et al., 2004; Matsui et al., 2007) while other ERP measures were barely studied in PD. It remained unclear whether the ERP components were altered in PD with hyposmia, especially the early ERP components reflecting visuospatial processing which was one of the mostly impaired cognitive domains in PD.

The delayed match to sample (DMS) task, one of the most popular tasks during ERP records in PD patients (Seer et al., 2016), was used in our study to test working memory which related to several cognitive abilities including storage capacity, retrieval strategies (Unsworth and Engle, 2007) and visuospatial attention (Bleckley et al., 2003; Giuliano et al., 2014). And Chinese characters applied in our DMS task were more acceptable for Chinese participants and more dependable on visual working memory (Opitz et al., 2014). What's more, we focused on retrieval epoch rather than encoding epoch in view of impaired retrieval process and reserved encoding ability of memory deficit in PD (Mahurin et al., 1993).

To our knowledge, there was no research focusing on cognitive ERP measures in PD patients with hyposmia. The aim of this study was to improve our understanding of the role of hyposmia in cognitive impairment in PD using the ERP technique with DMS task. Our study investigated difference in ERP components and its association with cognitive domains between PD patients with and without hyposmia.

\section{MATERIALS AND METHODS}

\section{Subject}

Idiopathic PD patients aging from 50 to 80 were recruited from movement disorder clinic of the Department of Neurology, Ruijin Hospital affiliated to Shanghai Jiao Tong University School of Medicine from September 2016 to October 2017. PD was diagnosed according to the clinical Movement Disorder Society (MDS) diagnostic criteria (Postuma et al., 2015) by senior Movement Disorder Specialist. Exclusion criteria included history of head injury, stroke, psychiatric disorder, poor vision, nasal and paranasal disease or other factors affecting olfactory function. Patients with less than 6 years of education or Mini-Mental State Examination (MMSE) score less than 24 were also excluded.

Finally 43 PD patients were enrolled in our study. Hyposmia was defined with score of Sniffin' Sticks test-16 (SS-16) (Burghart Messtechnik, Wedel, Germany) less than 8.3 (Chen et al., 2012) which was consistent with previous researches in our department (Chen et al., 2015). There were 24 subjects in PD with hyposmia group and 19 in PD without hyposmia group. All participants were informed of the research protocol and this study was carried out in accordance with the recommendations of Ethical Review of Biomedical Research Involving Human Subjects by China's Ministry of Health with written informed consent from all subjects. The protocol was approved by Ethics Committee of Ruijin Hospital affiliated to Shanghai Jiao Tong University School of Medicine, Shanghai, China. 


\section{Neuropsychological Assessment}

Demographic characters including age, sex, education years and disease duration were recorded (Table 1). The disease progression was assessed by modified Hoehn and Yahr (H-Y) scale (Hoehn and Yahr, 1967) and levodopa equivalent dose (LED) which was calculated as reported (Tomlinson et al., 2010). Motor subtypes were divided into tremor dominant type and non-tremor dominant type. Montreal Cognitive Assessment (MOCA) Beijing version was used to evaluate cognitive performance (Yu et al., 2012). Night sleep quality was assessed by Parkinson's disease Sleep Scale (PDSS) (Wang et al., 2008). Rapid Eye Movement Sleep Behavior Disorder Questionnaire-Hong Kong (RBD-HK) (Shen et al., 2014) and Epworth Sleepiness Scale (ESS) (Kumar et al., 2003) were used to evaluate RBD and excessive daytime sleepiness (EDS). The anxiety symptoms were evaluated by Hamilton Anxiety Rating

TABLE 1 | Demographics and clinical characteristics in PD with and without hyposmia groups (mean \pm standard deviation).

\begin{tabular}{lccc}
\hline & $\begin{array}{c}\text { PD without hyposmia } \\
(\boldsymbol{n}=\mathbf{1 9})\end{array}$ & $\begin{array}{c}\text { PD with hyposmia } \\
(\boldsymbol{n}=\mathbf{2 4})\end{array}$ & $\boldsymbol{P}$ \\
\hline Age (years) & $62.37 \pm 6.19$ & $64.71 \pm 5.89$ & 0.213 \\
Sex (M/n) & $8 / 19$ & $11 / 24$ & 0.807 \\
Disease & $5.31 \pm 5.46$ & $5.63 \pm 4.29$ & 0.835 \\
duration (years) & & & \\
LED (mg) & $269.66 \pm 260.57$ & $280.65 \pm 174.76$ & 0.871 \\
Education & $12.05 \pm 2.30$ & $12.92 \pm 2.50$ & 0.250 \\
(years) & & & \\
H-Y stage & $1.68 \pm 0.71$ & $1.75 \pm 0.59$ & 0.742 \\
Motor subtype & $7 / 19$ & $11 / 22$ & 0.553 \\
(tremor type/n) & & & \\
SS-16 & $10.58 \pm 1.64$ & $6.00 \pm 2.02$ & 0.000 \\
PDSS & $126.84 \pm 20.61$ & $117.75 \pm 16.59$ & 0.116 \\
RBD-HK & $14.84 \pm 17.58$ & $13.13 \pm 14.00$ & 0.723 \\
ESS & $5.95 \pm 4.76$ & $5.71 \pm 4.84$ & 0.872 \\
HAMD-17 & $3.74 \pm 3.96$ & $3.83 \pm 4.06$ & 0.938 \\
HAMA & $5.16 \pm 4.80$ & $3.75 \pm 3.18$ & 0.255 \\
MOCA & $25.79 \pm 1.93$ & $26.79 \pm 2.64$ & 0.173 \\
MMSE & $28.68 \pm 1.46$ & $28.46 \pm 2.06$ & 0.688
\end{tabular}

Scale (HAMA) (Thompson, 2015) and 17-item Hamilton Rating Scale for Depression (HAMD-17) (Hamilton, 1960) was used for depression screening.

\section{The Delayed Match to Sample Task}

Each participant was required to participate in a practice block before the test block during the DMS task (Figure 1). In each trial, after a single of " + " in the screen of $500 \mathrm{~ms}$, a set of six visually similar and easily confused Chinese characters (sample stimulus) presented for $2000 \mathrm{~ms}$ which should be remembered by subjects. After a blank for $3000 \mathrm{~ms}$, a character (probe stimulus) demonstrated in the middle of the screen could either be a member of the previous set of characters (answer "yes") or be another similar-looking one (answer "no"). The character presented until a response before $2000 \mathrm{~ms}$ or until $2000 \mathrm{~ms}$ without response. Subjects were asked to answer "yes" by pressing the button of "1" or "no" by pressing the button of " 2 " on a keyboard as soon as the response was ensured. The "yes" and "no" answers were randomized and 50\% of the correct answers were "yes" and the rest was "no" in each block. The inter-trial interval was $5000 \mathrm{~ms}$. Patients were allowed to begin the test until the accuracy of the practice block was higher than $50 \%$. Each test contained 100 trials. This DMS task was programmed by E-Prime 2.0 software (Psychology Software Tools, Inc., Pittsburgh, PA, United States) in which reaction time and accuracy of each subject were also recorded. The retrieval period of our study was defined from $200 \mathrm{~ms}$ before probe stimulus onset to $1000 \mathrm{~ms}$ after.

\section{EEG Recordings and Data Analysis}

ERP was recorded from $32 \mathrm{Ag}$ - $\mathrm{AgCl}$ electrodes (Fp1, Fp2, F3, F4, C3, C4, P3, P4, O1, O2, F7, F8, T7, T8, P7, P8, Fz, FCz, Cz, Pz, FC1, FC2, CP1, CP2, FC5, FC6, CP5, CP6, FT9, FT10, TP9, TP10) which were placed according to the international 10-20 system with a 32-channel amplifier (BrainAmp by Brain Products, Munich, Germany).

All channels were digitized at $500 \mathrm{~Hz}$ with $200 \mathrm{~ms}$-long prestimulus baseline used for baseline correction. The filter bandpass was $0.1-70 \mathrm{~Hz}$. Electro-oculogram (EOG) was also recorded to correct ocular artifacts. A semi-automatic check was used in artifact rejection. Trials were rejected if EEG voltage step was

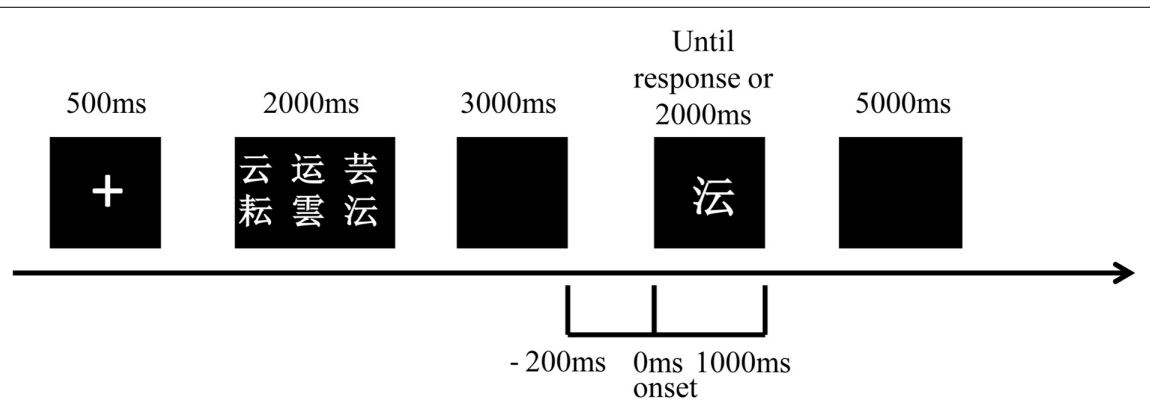

FIGURE 1 | Schematic representation of the visual DMS task used for ERP recordings. A set of six Chinese characters (sample stimulus) was displayed after a single of "+" and was required to be remembered by participants. After a blank for $3000 \mathrm{~ms}$, a character (probe stimulus) presented for 2000 ms and the answer of "yes" or "no" was required to decide whether this character was a member of the set of characters or not. The inter-trial interval was 5000 ms. The retrieval period is from $200 \mathrm{~ms}$ before probe stimulus onset to $1000 \mathrm{~ms}$ after. 
higher than $50 \mu \mathrm{V} / \mathrm{ms}$ or if difference of values in intervals was higher than $200 \mu \mathrm{V}$ or lower than $0.5 \mu \mathrm{V}$. The mean rejection rate for all participants was higher than $1 \%$. ERPs were based on correct trials and data not rejected for artifacts.

The five ERP components including N1, N2, P1, P2, and P300 were analyzed in reference of early studies, meanwhile, electrodes with maximal amplitude were chosen, which was consistent with the previous report of our center using the same paradigm (Li et al., 2016). N1 amplitude was measured as the maximal negative peak within the latency window of $150-210 \mathrm{~ms}$ on P7, P8 electrodes. P1 amplitude was identified at the most positive peak on O1, O2, P7 and P8 within 84-140 ms after the stimulus onset. $\mathrm{N} 2$ amplitude was considered at the maximal negative peak on F3, F4, C3, C4, CZ, FZ and FCz within the latency window of 230-300 ms. P2 amplitude was identified at the maximal positive peak on FC1, FC2, CZ, FZ and FCz within the latency window of 150-250 ms. The P300component amplitude was analyzed as the maximal positive peak within $250-450 \mathrm{~ms}$ on $\mathrm{O} 1, \mathrm{O} 2$ and $\mathrm{Pz}$ electrodes. The N1P2 amplitude was summed absolute amplitude of the $\mathrm{N} 1$ and $\mathrm{P} 2$ peaks on $\mathrm{P} 7, \mathrm{P} 8, \mathrm{FC} 1, \mathrm{FC} 2, \mathrm{CZ}, \mathrm{FZ}$ and $\mathrm{FCz}$ electrodes. The N1P2 amplitude divided by average noise level yielded the signal-to-noise $(\mathrm{S} / \mathrm{N})$ ratio.

\section{Statistical Analysis}

All statistical analysis was performed by SPSS 19.0 (IBM, Armonk, NY). Demographic characteristics and clinical variables were compared between the two groups using the independent sample $t$-test or chi square analysis. The Spearman's correlation was analyzed between each ERP component parameter and MOCA item score. The association between SS-16 and MOCA item score was also analyzed by Spearman's correlation. All ERP components parameters were compared between the two groups using independent Mann-Whitney $U$-test. All tests were 2-tailed and $P$-values $<0.05$ were considered statistically significant.

\section{RESULTS}

\section{Demographics and Clinical Characteristics}

There was no significant group difference in demographic characters including age, sex, and education degree. No significant difference was revealed in disease duration, H-Y stage, tremor subtype and LED between PD with and without hyposmia. Questionnaire results of sleep disorder (PDSS, RBDHK and ESS) as well as anxiety (HAMA) and depression (HAMD-17) were similar between the two groups. No significant difference was found in total score of MMSE or MOCA (Table 1).

\section{Task Performance and Brain Activity}

There was no significant difference between PD patients with and without hyposmia regarding number of valid trials, reaction time and accuracy. No significant difference was found in parameters of ERP components including N1, N2, P1, P2 and P300 between the two groups (Table 2 and Figure 2). Notably, the N1P2 amplitude did not differ between the two groups. And the mean
TABLE 2 | Task performance and parameters of ERP components in PD with and without hyposmia groups (mean \pm standard deviation).

\begin{tabular}{|c|c|c|c|}
\hline & $\begin{array}{l}\text { PD without hyposmia } \\
\qquad(n=19)\end{array}$ & $\begin{array}{l}\text { PD with hyposmia } \\
\qquad(n=24)\end{array}$ & $P$ \\
\hline $\begin{array}{l}\text { Number of valid } \\
\text { trials }\end{array}$ & 1625 & 2048 & 0.954 \\
\hline Accuracy (\%) & $89.79 \pm 8.38$ & $88.21 \pm 19.39$ & 0.742 \\
\hline $\begin{array}{l}\text { Reaction time } \\
\text { (s) }\end{array}$ & $1590.49 \pm 226.51$ & $1549.82 \pm 246.54$ & 0.581 \\
\hline N1 latency (ms) & $162.16 \pm 12.05$ & $158.54 \pm 7.75$ & 0.265 \\
\hline $\begin{array}{l}\text { N1 amplitude } \\
(\mu \mathrm{V})\end{array}$ & $-6.05 \pm 3.30$ & $-7.17 \pm 3.92$ & 0.391 \\
\hline N2 latency (ms) & $261.98 \pm 15.06$ & $262.42 \pm 21.12$ & 0.940 \\
\hline $\begin{array}{l}\text { N2amplitude } \\
(\mu \mathrm{V})\end{array}$ & $-1.94 \pm 3.29$ & $-1.89 \pm 2.88$ & 0.964 \\
\hline P1 latency (ms) & $98.58 \pm 8.00$ & $99.83 \pm 9.30$ & 0.643 \\
\hline $\begin{array}{l}\text { P1 amplitude } \\
(\mu \mathrm{V})\end{array}$ & $4.98 \pm 3.31$ & $4.77 \pm 2.75$ & 0.818 \\
\hline P2 latency (ms) & $169.43 \pm 20.04$ & $173.35 \pm 28.51$ & 0.615 \\
\hline $\begin{array}{l}\text { P2 amplitude } \\
(\mu \mathrm{V})\end{array}$ & $5.39 \pm 3.97$ & $5.63 \pm 2.63$ & 0.807 \\
\hline P3 latency (ms) & $372.25 \pm 54.80$ & $391.53 \pm 46.93$ & 0.221 \\
\hline $\begin{array}{l}\text { P3 amplitude } \\
(\mu \mathrm{V})\end{array}$ & $5.03 \pm 2.96$ & $6.40 \pm 4.01$ & 0.220 \\
\hline $\begin{array}{l}\text { N1P2 } \\
\text { amplitude }(\mu \mathrm{V})\end{array}$ & $8.28 \pm 4.83$ & $8.23 \pm 3.19$ & 0.509 \\
\hline $\mathrm{S} / \mathrm{N}$ & $4.90 \pm 2.32$ & $5.17 \pm 2.53$ & 0.826 \\
\hline
\end{tabular}

$\mathrm{S} / \mathrm{N}$ ratio was 4.90 in the normosmic group and 5.17 in the hyposmic group and no difference was found between the two groups (Table 2).

\section{Correlation Between ERPs and Cognitive Ability}

Correlation was analyzed between ERP component parameter and cognitive performance in all participants (Tables 3, 4). N1 latency was significantly negatively related to visuospatialexecutive item score of MOCA $\left(r_{\mathrm{s}}=-0.381, P=0.012\right)$. Among three visuospatial-executive tests, a significant negative correlation between $\mathrm{N} 1$ latency and test score was found in cube copy test $\left(r_{\mathrm{s}}=-0.401, P=0.008\right)$ and clock drawing $\left(r_{\mathrm{s}}=-0.451, P=0.002\right)$ but not in trail making test. What's more, a significant positive correlation was found between P1 amplitude and language item score of MOCA $\left(r_{\mathrm{s}}=0.302, P=0.049\right)$. There was no correlation between MOCA total score and N1 or P1 parameters. Parameters of other ERP components including N2, P2 and P300 were not found significantly associated with MOCA score or MOCA item score. Then the correlations between N1 latency and P1 amplitude and visuospatial-executive and language item score were also analyzed in separate groups (Table 5). There was a significant negative correlation between N1 latency and visuospatial-executive item score within the normosmic group $\left(r_{\mathrm{s}}=-0.619, P=0.005\right)$ but not within the hyposmic group. Among three visuospatial tests, a significant negative correlation with N1 latency was found in both cube copy test $\left(r_{\mathrm{s}}=-0.585\right.$, 


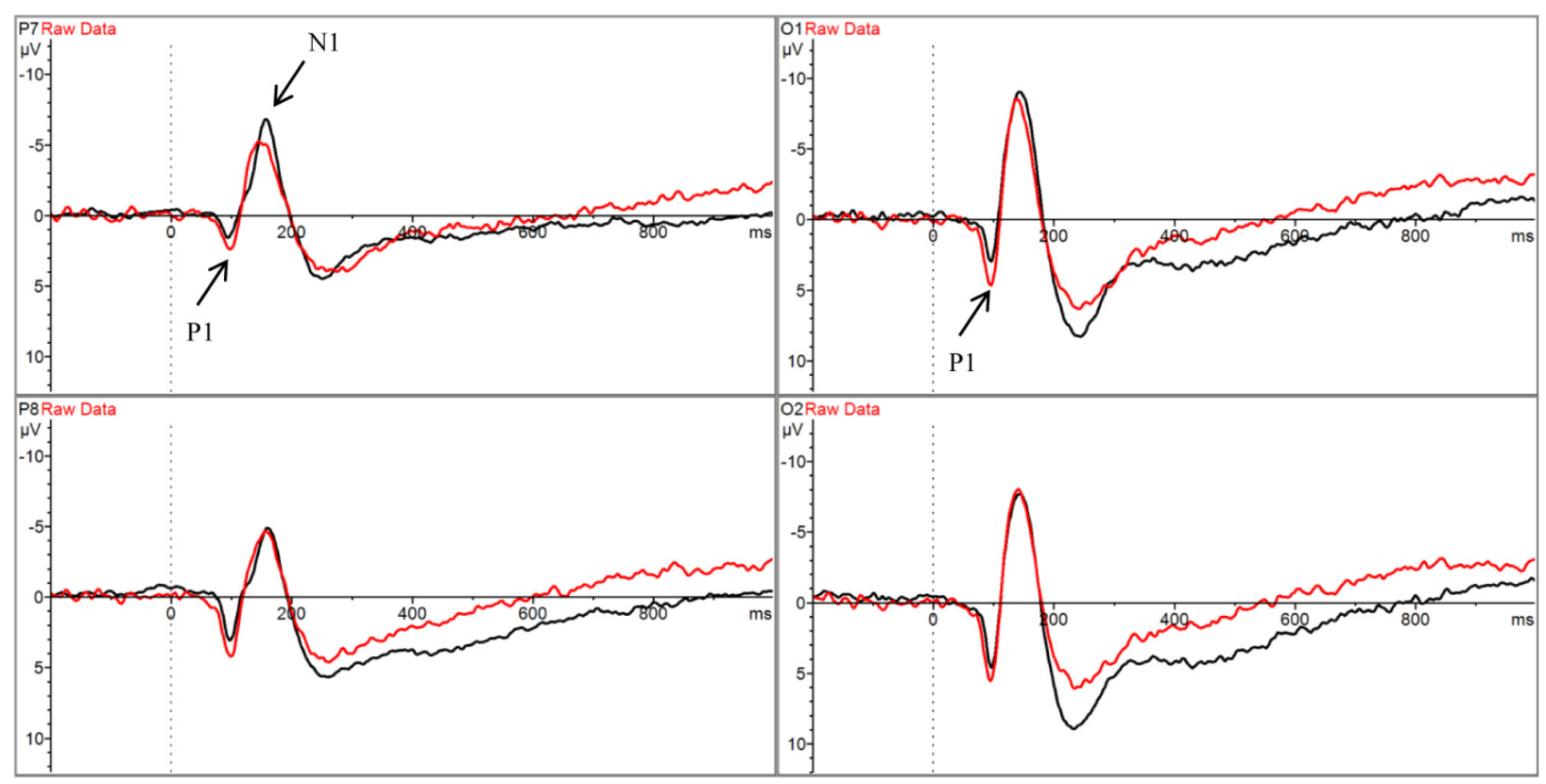

PD with hyposmia

PD without hyposmia

FIGURE 2 | Grand average ERP waveform in PD with hyposmia (black line) and PD without hyposmia (red line) during retrieval period in DMS task. Latency was recorded in milliseconds with stimulus onset at 0 . Amplitude was recorded in micro-voltage.

$P=0.008)$ and clock drawing test $\left(r_{\mathrm{s}}=-0.523, P=0.022\right)$ within the normosmic group and only in clock drawing test ( $\left.r_{\mathrm{s}}=-0.413, P=0.045\right)$ within the hyposmic group. In addition, a significant correlation between P1 amplitude and language item score was found within the normosmic group ( $r_{\mathrm{s}}=0.537, P=0.018$ ) but not within the hyposmic group. Furthermore, SS-16 score was not found to be significantly associated with visuospatial-executive or language item score of MOCA (Table 6).

Correlation was also analyzed between parameters of $\mathrm{N} 1$ and P1 components and clinical characteristics in all subjects (Table 7). N1 latency was found to be significantly negatively related to education degree $\left(r_{\mathrm{s}}=-0.326, P=0.033\right)$. There was a significant positive correlation between HAMA score and N1 amplitude $\left(r_{\mathrm{s}}=0.360, P=0.018\right)$. No significant correlation was found between other component parameters and clinical characteristics.

\section{DISCUSSION}

To our knowledge, our study was the first to compare cognitive ERP measures between PD patients with and without hyposmia. No significant difference in ERP performance was found between the two groups. Among all subjects, N1 latency was significantly negatively related to visuospatial-executive function and there was a significant positive correlation between P1 amplitude and
TABLE 3 | Correlation analysis between ERP latency and MOCA score in all participants (correlation coefficient $r_{\mathrm{s}}$ ).

\begin{tabular}{lrrrrr}
\hline & N1 & N2 & P1 & P2 & P3 \\
\hline Visuospatial-executive & $-0.381^{*}$ & 0.085 & -0.068 & -0.104 & 0.121 \\
$\begin{array}{l}\text { Visuospatial-executive } \\
\text { (trail making test) }\end{array}$ & -0.105 & 0.107 & -0.067 & 0.103 & 0.079 \\
Visuospatial-executive & $-0.401^{* *}$ & 0.178 & -0.009 & 0.000 & 0.094 \\
(cube copy) & & & & & \\
Visuospatial-executive & $-0.451^{*}$ & 0.021 & -0.037 & -0.207 & 0.069 \\
(clock drawing) & & & & & \\
Naming & -0.044 & 0.084 & -0.038 & -0.191 & 0.164 \\
Attention & 0.092 & -0.131 & 0.000 & 0.041 & -0.023 \\
Language & 0.101 & -0.027 & 0.036 & 0.020 & 0.237 \\
Abstraction & -0.232 & -0.125 & 0.051 & -0.088 & 0.193 \\
Delayed Recall & 0.034 & 0.199 & -0.003 & 0.136 & 0.236 \\
Orientation & -0.206 & 0.080 & -0.004 & -0.067 & -0.098 \\
Total score & -0.217 & 0.100 & -0.024 & -0.003 & 0.223 \\
\hline$* P<0.05 ; * * P 0.01$. & & & & &
\end{tabular}

language ability. Within the normosmic group, N1 and P1 also associated with visuospatial-executive and language functions while a significant correlation was only found between N1 latency and clock drawing test performance rather than visuospatialexecutive item score in the hyposmic group. What's more, odor identification ability was not found to be significantly related to visuospatial-executive function or language ability. 
TABLE 4 | Correlation analysis between ERP amplitude and MOCA score in all participants (correlation coefficient $r_{\mathrm{S}}$ ).

\begin{tabular}{lrrrrr}
\hline & N1 & N2 & P1 & P2 & P3 \\
\hline Visuospatial-executive & 0.010 & -0.077 & -0.185 & -0.288 & -0.147 \\
$\begin{array}{l}\text { Visuospatial-executive } \\
\text { (trail making test) }\end{array}$ & -0.100 & -0.079 & -0.234 & -0.188 & -0.205 \\
Visuospatial-executive & 0.053 & 0.193 & -0.094 & -0.164 & -0.228 \\
$\begin{array}{l}\text { (cube copy) } \\
\text { Visuospatial-executive }\end{array}$ & 0.035 & -0.067 & -0.018 & -0.179 & -0.016 \\
(clock drawing) & & & & & \\
Naming & 0.059 & -0.206 & 0.193 & 0.037 & 0.200 \\
Attention & 0.053 & 0.003 & -0.161 & -0.034 & 0.081 \\
Language & -0.195 & -0.120 & $0.302^{*}$ & 0.231 & 0.075 \\
Abstraction & -0.140 & -0.069 & -0.033 & -0.038 & 0.222 \\
Delayed Recall & 0.142 & 0.226 & -0.114 & 0.088 & 0.040 \\
Orientation & -0.214 & 0.071 & -0.089 & 0.169 & -0.169 \\
Total score & 0.005 & 0.089 & -0.053 & 0.020 & 0.002 \\
\hline
\end{tabular}

$* P<0.05$.

TABLE 5 | Correlation analysis between parameters of ERP components (N1 and $\mathrm{P1}$ ) and MOCA item (visuospatial-executive and language items) score in PD with and without hyposmia groups (correlation coefficient $r_{\mathrm{S}}$ ).

\begin{tabular}{|c|c|c|c|c|}
\hline & \multicolumn{2}{|c|}{ PD without hyposmia } & \multicolumn{2}{|c|}{ PD with hyposmia } \\
\hline & N1 latency & P1 amplitude & N1 latency & P1 amplitude \\
\hline Visuospatial- executive & $-0.619^{* *}$ & -0.182 & -0.161 & -0.121 \\
\hline $\begin{array}{l}\text { Visuospatial- executive } \\
\text { (trail making test) }\end{array}$ & -0.303 & -0.207 & 0.119 & -0.153 \\
\hline $\begin{array}{l}\text { Visuospatial- executive } \\
\text { (cube copy) }\end{array}$ & $-0.585^{* *}$ & -0.184 & -0.153 & 0.044 \\
\hline $\begin{array}{l}\text { Visuospatial- Executive } \\
\text { (clock drawing) }\end{array}$ & $-0.523^{*}$ & 0.040 & $-0.413^{*}$ & -0.059 \\
\hline Language & 0.104 & $0.537^{*}$ & 0.098 & 0.032 \\
\hline
\end{tabular}

$* P<0.05 ; * * P<0.01$.

TABLE 6 | Correlation analysis between SS-16 score and MOCA item (visuospatial-executive and language items) score in all participants (correlation coefficient $r_{\mathrm{s}}$ ).

\begin{tabular}{lc}
\hline & SS-16 \\
\hline Visuospatial-executive & -0.088 \\
Visuospatial-executive (trail making test) & -0.034 \\
Visuospatial-executive (cube copy) & -0.118 \\
Visuospatial-executive (clock drawing) & -0.125 \\
Language & -0.201 \\
Total score & -0.099 \\
\hline
\end{tabular}

Hyposmia is an important clinical biomarker of PD and there are four subtypes of olfactory dysfunction reported in PD including impairment of odor identification, odor discrimination, odor threshold detection and odor recognition memory (Mesholam et al., 1998; Haehner et al., 2009). Odor identification deficit is the most prevalent form of hyposmia and is tested mainly by University of Pennsylvania Smell Identification Test (UPSIT) in PD patients (Doty et al.,
TABLE 7 | Correlation analysis between parameters of ERP components (N1 and $\mathrm{P} 1$ ) and clinical characteristics in all participants (correlation coefficient $r_{\mathrm{s}}$ ).

\begin{tabular}{lrcrc}
\hline & N1 latency & N1 amplitude & P1 latency & P1 amplitude \\
\hline Age (years) & -0.177 & 0.143 & 0.058 & 0.175 \\
Disease duration & 0.037 & -0.105 & 0.203 & 0.076 \\
(years) & & & & \\
Education degree & $-0.326 *$ & -0.091 & -0.232 & -0.058 \\
(years) & & & & \\
LED (mg) & 0.086 & 0.028 & 0.162 & 0.134 \\
ESS & 0.089 & 0.041 & 0.287 & 0.086 \\
PDSS & 0.260 & -0.128 & -0.046 & 0.226 \\
RBD-HK & -0.047 & 0.163 & 0.003 & 0.191 \\
H-Y stage & 0.091 & 0.149 & 0.236 & 0.051 \\
HAMA & 0.127 & $0.360 *$ & 0.035 & 0.047 \\
HAMD-17 & -0.074 & 0.191 & -0.045 & -0.095 \\
\hline * $<0.05$ & & & &
\end{tabular}

1988) and SS-16 (Haehner et al., 2009) which was used in our study considering its advantage in cross-cultural application.

Previous studies found a significant association between hyposmia and cognitive impairment in PD patients. The percentage of cognitive decline was significantly higher in PD patients with hyposmia than those without (Bohnen et al., 2010; Stephenson et al., 2010; Morley et al., 2011; Parrao et al., 2012). And olfactory dysfunction significantly increased the risk of dementia in $\mathrm{PD}$ patients regardless the cognitive functions at baseline according to a 10-year follow up study (Domellof et al., 2017). The underlying mechanism remained unclear. The olfactory bulb was known to be one of the onset sites with appearance of Lewy body pathology during PD progression in Braak's stage (Braak et al., 2003). And over expression of alphasynuclein in the olfactory bulb was reported to initiate hyposmia and other prodromal symptoms of PD in rats (Niu et al., 2018). A hypothesis was proposed that severe olfactory dysfunction might be related to abundant cortical Lewy body deposition (Morley et al., 2011) which was found to be associated with PD dementia (Tsuboi et al., 2007). What's more, the relation between hyposmia and cognitive impairment could also be explained by cholinergic denervation of the limbic archicortex (Bohnen et al., 2010) and dopaminergic denervation of the hippocampus (Bohnen et al., 2008). In our study, no significant difference was found in MMSE or MOCA score between PD with and without hyposmia, which may be associated with limits of our sample size and sensitivities of MMSE and MOCA in cognitive impairment of PD patients (Burdick et al., 2014; Wyman-Chick et al., 2017). Interestingly, no significant difference was found in parameters of ERP components including N1, N2, P1, P2 and P300 recorded during DMS tasks between these two groups. Therefore, the association between cognitive domains and ERP measures in PD with and without hyposmia was important to be investigated.

$\mathrm{N} 1$ and P1 were found to respectively associate with visuospatial-executive functions and language in our study while no significant correlation was found between the other components including P2, N2, and P300 and specific cognitive 
functions. N1 and P1 are the earliest components in ERP representing visual sensory input. The generator of visual N1 is probably located in lateral extrastriate cortex (Gomez Gonzalez et al., 1994), dorsal occipito-parietal and ventral occipitotemporal areas (Yamazaki et al., 2000) and the visual P1 may be generated in fusiform gyrus (Heinze et al., 1994). N1 and P1 were reported to be associated with visual perceptual processing (Vogel and Luck, 2000; Taylor, 2002) especially in visuospatial attention according to previous reports (Naatanen and Picton, 1987; Luck et al., 1990). Luck et al. (1990) found that N1 was related to attention orienting to relevant stimulus and P1 possibly reflected a facilitation of early sensory processing with attention already focused. On the other hand, several studies demonstrated the role of $\mathrm{N} 1$ and $\mathrm{P} 1$ in language perception. Selpien et al. (2015) found that N1 and P1 were significantly related to language lateralization. In an auditory ERP study, the association between P1 amplitude and attention was found in linguistic probes stimulation but not in non-linguistic probes (Giuliano et al., 2014).

Abnormal N1 and P1 components in PD patients were observed in several studies. Wright et al. (1996) found attenuated N1 amplitudes during Auditory Oddball Task in 17 PD patients. However, enlarged N1 and P1 amplitudes as well as shortened $\mathrm{N} 1$ latency in $34 \mathrm{PD}$ patients were demonstrated in a visual ERP study with oddball and S1-S2 tasks (Li et al., 2003). The discrepancy may be explained by the differences of tasks used in ERP, sample size and analytical approach of component parameters. Unfortunately, the clinical significance of $\mathrm{N} 1$ and P1 components in PD patients was investigated in very few studies. Wang et al. (2001) reported the association between $\mathrm{N} 1$ and cognitive visual processing in $\mathrm{PD}$ patients evidenced by its correlation with the regional cerebral blood flow. Consistent to previous studies, our study found a significant negative correlation between N1 latency and visuospatial-executive function in PD patients and P1 amplitude was significantly positively related to language ability. Therefore N1 latency and P1 amplitude during DMS task were possible to be used for assessing visuospatial-executive functions and language abilities in PD patients. Interestingly, the significant association between these cognitive functions and $\mathrm{N} 1$ and $\mathrm{P} 1$ components were also found in the normosmic group while in the hyposmic group, there was only a significant correlation between N1 and clock drawing test score rather than visuospatial-executive item score. To explain difference in clinical significance of N1 and P1 components between the two groups, one hypothesis could be that there exists different cognitive processing between these normosmic and hyposmic participants. Increasing evidence shows that PD with and without hyposmia may be two subtypes of PD patients. Lee et al. (2015) found that normosmic PD represented a unique clinical phenotype with a more benign with fewer motor deficits and higher dopamine transporter activity. On the other hand, hyposmia in PD was reported to be associated with tremor-dominant type (Iijima et al., 2011), SNCA rs11931074 and specific non-motor symptoms such as RBD and chronic constipation (Chen et al., 2015). From the aspect of cognition, a good olfactory performance in PD was found to compensate gray matter volume loss (Lee et al., 2014) and there were cholinergic and dopaminergic denervations in PD with hyposmia (Bohnen et al., 2008, 2010). Therefore, some components underlying a cognitive process such as visuospatialexecutive and language functions could be different between PD with and without hyposmia.

The impaired cognitive domains in PD with hyposmia were under debate. Bohnen et al. (2010) found that UPSIT scores were significantly related to episodic verbal learning in 58 PD patients but not other cognitive performance including visuospatial function, visual non-verbal memory, attention and executive function. However, poorer memory and executive functions were reported to be associated with worse olfactory identification in other studies (Morley et al., 2011; Parrao et al., 2012). Our study demonstrated no significant association between SS-16 score and visuospatial-executive or language item score of MOCA in PD patients. The discrepancy is possibly explained by difference in sample size and neurocognitive test batteries which may be avoided by using ERP measures.

There were several limitations of our study. A larger sample size is expected in further studies to confirm our results. In addition, only MOCA with relatively high reliability and validation reported (Nie et al., 2012) was used for cognitive assessment in our study as the psychometric properties of neurocognitive test batteries were rarely studied in Chinese PD patients. What's more, only odor identification was tested which was the mostly impaired olfactory function in PD (Doty et al., 1988). However, in view of possibly different mechanisms underlying four subtypes of hyposmia (Kareken et al., 2003; Hedner et al., 2010), the association between cognitive domains and the other three olfactory functions in PD warrants further investigation.

In conclusion, our study compared ERP measures recorded during DMS tasks at retrieval period and its association with cognitive domains between PD patients with and without hyposmia. No significant difference in ERP components was found between the two groups in the present study. Among all participants, N1 latency was significantly negatively related to visuospatial-executive functions and there was also a significantly positive correlation between P1 amplitude and language ability. In PD without hyposmia, N1 latency and P1 amplitude were respectively associated with visuospatial-executive and language functions while in PD with hyposmia, only a significant correlation was found between N1 latency and clock drawing test performance rather than visuospatial-executive item score in MOCA. What's more, no significant association was found between odor identification ability and visuospatial-executive or language item score of MOCA.

\section{AUTHOR CONTRIBUTIONS}

All the authors cooperated and contributed to the design and plan of the present study. Y-QL, S-SC, J-JD, GL, Y-XH, P-CZ, YF, PH, and CG performed the research. S-DC was in charge of clinical diagnosis. Y-QL and B-YL analyzed the data. Y-QL wrote the original article. S-DC and B-YL were in charge of manuscript verifying. 


\section{FUNDING}

This work was supported by grants from the National Natural Science Foundation of China (Nos. 81430022 and 81771374).

\section{REFERENCES}

Attems, J., Lintner, F., and Jellinger, K. A. (2005). Olfactory involvement in aging and Alzheimer's disease: an autopsy study. J. Alzheimers Dis. 7, 149-157; discussion 173-180. doi: 10.3233/JAD-2005-7208

Bleckley, M. K., Durso, F. T., Crutchfield, J. M., Engle, R. W., and Khanna, M. M. (2003). Individual differences in working memory capacity predict visual attention allocation. Psychon. Bull. Rev. 10, 884-889. doi: 10.3758/BF03196548

Boesveldt, S., Verbaan, D., Knol, D. L., Visser, M., Van Rooden, S. M., Van Hilten, J. J., et al. (2008). A comparative study of odor identification and odor discrimination deficits in Parkinson's disease. Mov. Disord. 23, 1984-1990. doi: $10.1002 / \mathrm{mds} .22155$

Bohnen, N. I., Gedela, S., Herath, P., Constantine, G. M., and Moore, R. Y. (2008). Selective hyposmia in Parkinson disease: association with hippocampal dopamine activity. Neurosci. Lett. 447, 12-16. doi: 10.1016/j.neulet.2008.09.070

Bohnen, N. I., Muller, M. L., Kotagal, V., Koeppe, R. A., Kilbourn, M. A., Albin, R. L., et al. (2010). Olfactory dysfunction, central cholinergic integrity and cognitive impairment in Parkinson's disease. Brain 133, 1747-1754. doi: 10. 1093/brain/awq079

Braak, H., Del Tredici, K., Rub, U., De Vos, R. A., Jansen Steur, E. N., and Braak, E. (2003). Staging of brain pathology related to sporadic Parkinson's disease. Neurobiol. Aging 24, 197-211. doi: 10.1016/S0197-4580(02)00065-9

Burdick, D. J., Cholerton, B., Watson, G. S., Siderowf, A., Trojanowski, J. Q., Weintraub, D., et al. (2014). People with Parkinson's disease and normal MMSE score have a broad range of cognitive performance. Mov. Disord. 29, 1258-1264. doi: $10.1002 / \mathrm{mds} .25924$

Chen, W., Chen, S., Kang, W. Y., Li, B., Xu, Z. M., Xiao, Q., et al. (2012). Application of odor identification test in Parkinson's disease in China: a matched case-control study. J. Neurol. Sci. 316, 47-50. doi: 10.1016/j.jns.2012. 01.033

Chen, W., Kang, W. Y., Chen, S., Wang, Y., Xiao, Q., Wang, G., et al. (2015). Hyposmia correlates with SNCA variant and non-motor symptoms in Chinese patients with Parkinson's disease. Parkinsonism Relat. Disord. 21, 610-614. doi: 10.1016/j.parkreldis.2015.03.021

Domellof, M. E., Lundin, K. F., Edstrom, M., and Forsgren, L. (2017). Olfactory dysfunction and dementia in newly diagnosed patients with Parkinson's disease. Parkinsonism Relat. Disord. 38, 41-47. doi: 10.1016/j.parkreldis.2017.02.017

Doty, R. L. (2012). Olfaction in Parkinson's disease and related disorders. Neurobiol. Dis. 46, 527-552. doi: 10.1016/j.nbd.2011.10.026

Doty, R. L., Deems, D. A., and Stellar, S. (1988). Olfactory dysfunction in parkinsonism: a general deficit unrelated to neurologic signs, disease stage, or disease duration. Neurology 38, 1237-1244. doi: 10.1212/WNL.38.8.1237

Duda, J. E. (2010). Olfactory system pathology as a model of Lewy neurodegenerative disease. J. Neurol. Sci. 289, 49-54. doi: 10.1016/j.jns. 2009.08.042

Giuliano, R. J., Karns, C. M., Neville, H. J., and Hillyard, S. A. (2014). Early auditory evoked potential is modulated by selective attention and related to individual differences in visual working memory capacity. J. Cogn. Neurosci. 26, 2682-2690. doi: 10.1162/jocn_a_00684

Gomez Gonzalez, C. M., Clark, V. P., Fan, S., Luck, S. J., and Hillyard, S. A. (1994). Sources of attention-sensitive visual event-related potentials. Brain Topogr. 7, 41-51. doi: 10.1007/BF01184836

Haehner, A., Boesveldt, S., Berendse, H. W., Mackay-Sim, A., Fleischmann, J., Silburn, P. A., et al. (2009). Prevalence of smell loss in Parkinson's diseasea multicenter study. Parkinsonism Relat. Disord. 15, 490-494. doi: 10.1016/j. parkreldis.2008.12.005

Hamilton, M. (1960). A rating scale for depression. J. Neurol. Neurosurg. Psychiatry 23, 56-62. doi: 10.1136/jnnp.23.1.56

Hawkes, C. H., Shephard, B. C., and Daniel, S. E. (1997). Olfactory dysfunction in Parkinson's disease. J. Neurol. Neurosurg. Psychiatry 62, 436-446. doi: 10.1136/ jnnp.62.5.436

\section{ACKNOWLEDGMENTS}

The authors thank the patients for their participation in the project.

Hedner, M., Larsson, M., Arnold, N., Zucco, G. M., and Hummel, T. (2010). Cognitive factors in odor detection, odor discrimination, and odor identification tasks. J. Clin. Exp. Neuropsychol. 32, 1062-1067. doi: 10.1080/ 13803391003683070

Heinze, H. J., Mangun, G. R., Burchert, W., Hinrichs, H., Scholz, M., Munte, T. F., et al. (1994). Combined spatial and temporal imaging of brain activity during visual selective attention in humans. Nature 372, 543-546. doi: 10.1038/ $372543 \mathrm{a} 0$

Hoehn, M. M., and Yahr, M. D. (1967). Parkinsonism: onset, progression and mortality. Neurology 17, 427-442. doi: 10.1212/WNL.17.5.427

Iijima, M., Kobayakawa, T., Saito, S., Osawa, M., Tsutsumi, Y., Hashimoto, S., et al. (2011). Differences in odor identification among clinical subtypes of Parkinson's disease. Eur. J. Neurol. 18, 425-429. doi: 10.1111/j.1468-1331.2010.03167.x

Kareken, D. A., Mosnik, D. M., Doty, R. L., Dzemidzic, M., and Hutchins, G. D. (2003). Functional anatomy of human odor sensation, discrimination, and identification in health and aging. Neuropsychology 17, 482-495. doi: 10.1037/ 0894-4105.17.3.482

Katsarou, Z., Bostantjopoulou, S., Kimiskidis, V., Rossopoulos, E., and Kazis, A. (2004). Auditory event-related potentials in Parkinson's disease in relation to cognitive ability. Percept. Mot. Skills 98, 1441-1448. doi: 10.2466/pms.98.3c. 1441-1448

Kumar, S., Bhatia, M., and Behari, M. (2003). Excessive daytime sleepiness in Parkinson's disease as assessed by Epworth Sleepiness Scale (ESS). Sleep Med. 4, 339-342. doi: 10.1016/S1389-9457(03)00105-9

Lee, D. H., Oh, J. S., Ham, J. H., Lee, J. J., Lee, I., Lee, P. H., et al. (2015). Is normosmic Parkinson disease a unique clinical phenotype? Neurology 85, 1270-1275. doi: 10.1212/WNL.0000000000001999

Lee, J. E., Cho, K. H., Ham, J. H., Song, S. K., Sohn, Y. H., and Lee, P. H. (2014). Olfactory performance acts as a cognitive reserve in non-demented patients with Parkinson's disease. Parkinsonism Relat. Disord. 20, 186-191. doi: 10.1016/ j.parkreldis.2013.10.024

Li, B.-Y., Tang, H.-D., and Chen, S.-D. (2016). Retrieval deficiency in brain activity of working memory in amnesic mild cognitive impairment patients: a brain event-related potentials study. Front. Aging Neurosci. 8:54. doi: 10.3389/fnagi. 2016.00054

Li, M., Kuroiwa, Y., Wang, L., Kamitani, T., Takahashi, T., Suzuki, Y., et al. (2003). Early sensory information processes are enhanced on visual oddball and S1-S2 tasks in Parkinson's disease: a visual event-related potentials study. Parkinsonism Relat. Disord. 9, 329-340. doi: 10.1016/S1353-8020(02)00094-9

Luck, S. J., Heinze, H. J., Mangun, G. R., and Hillyard, S. A. (1990). Visual eventrelated potentials index focused attention within bilateral stimulus arrays. II. Functional dissociation of $\mathrm{P} 1$ and $\mathrm{N} 1$ components. Electroencephalogr. Clin. Neurophysiol. 75, 528-542. doi: 10.1016/0013-4694(90)90139-B

Mahurin, R. K., Feher, E. P., Nance, M. L., Levy, J. K., and Pirozzolo, F. J. (1993). “Cognition in Parkinson's disease and related disorders," in Neuropsychology of Alzheimer's Disease and Other Dementias. New York, NY: Oxford University Press, 308-349.

Matsui, H., Nishinaka, K., Oda, M., Kubori, T., and Udaka, F. (2007). Auditory event-related potentials in Parkinson's disease: prominent correlation with attention. Parkinsonism Relat. Disord. 13, 394-398. doi: 10.1016/j.parkreldis. 2006.12.012

Mesholam, R. I., Moberg, P. J., Mahr, R. N., and Doty, R. L. (1998). Olfaction in neurodegenerative disease: a meta-analysis of olfactory functioning in Alzheimer's and Parkinson's diseases. Arch. Neurol. 55, 84-90. doi: 10.1001/ archneur.55.1.84

Morley, J. F., Weintraub, D., Mamikonyan, E., Moberg, P. J., Siderowf, A. D., and Duda, J. E. (2011). Olfactory dysfunction is associated with neuropsychiatric manifestations in Parkinson's disease. Mov. Disord. 26, 2051-2057. doi: 10.1002/ mds. 23792

Naatanen, R., and Picton, T. (1987). The N1 wave of the human electric and magnetic response to sound: a review and an analysis of the 
component structure. Psychophysiology 24, 375-425. doi: 10.1111/j.1469-8986. 1987.tb00311.x

Nie, K., Zhang, Y., Wang, L., Zhao, J., Huang, Z., Gan, R., et al. (2012). A pilot study of psychometric properties of the Beijing version of Montreal Cognitive Assessment in patients with idiopathic Parkinson's disease in China. J. Clin. Neurosci. 19, 1497-1500. doi: 10.1016/j.jocn.2011.11.039

Niu, H., Shen, L., Li, T., Ren, C., Ding, S., Wang, L., et al. (2018). Alphasynuclein overexpression in the olfactory bulb initiates prodromal symptoms and pathology of Parkinson's disease. Transl. Neurodegener. 7:25. doi: 10.1186/ s40035-018-0128-6

Opitz, B., Schneiders, J. A., Krick, C. M., and Mecklinger, A. (2014). Selective transfer of visual working memory training on Chinese character learning. Neuropsychologia 53, 1-11. doi: 10.1016/j.neuropsychologia.2013.10.017

Parrao, T., Chana, P., Venegas, P., Behrens, M. I., and Aylwin, M. L. (2012). Olfactory deficits and cognitive dysfunction in Parkinson's disease. Neurodegener. Dis. 10, 179-182. doi: 10.1159/000335915

Postuma, R. B., Berg, D., Stern, M., Poewe, W., Olanow, C. W., Oertel, W., et al. (2015). MDS clinical diagnostic criteria for Parkinson's disease. Mov. Disord. 30, 1591-1601. doi: 10.1002/mds.26424

Seer, C., Lange, F., Georgiev, D., Jahanshahi, M., and Kopp, B. (2016). Event-related potentials and cognition in Parkinson's disease: an integrative review. Neurosci. Biobehav. Rev. 71, 691-714. doi: 10.1016/j.neubiorev.2016.08.003

Selpien, H., Siebert, C., Genc, E., Beste, C., Faustmann, P. M., Gunturkun, O., et al. (2015). Left dominance for language perception starts in the extrastriate cortex: an ERP and sLORETA study. Behav. Brain Res. 291, 325-333. doi: 10.1016/j.bbr. 2015.05.050

Shen, S. S., Shen, Y., Xiong, K. P., Chen, J., Mao, C. J., Huang, J. Y., et al. (2014). Validation study of REM sleep behavior disorder questionnaire-Hong Kong (RBDQ-HK) in east China. Sleep Med. 15, 952-958. doi: 10.1016/j.sleep.2014. 03.020

Stephenson, R., Houghton, D., Sundarararjan, S., Doty, R. L., Stern, M., Xie, S. X., et al. (2010). Odor identification deficits are associated with increased risk of neuropsychiatric complications in patients with Parkinson's disease. Mov. Disord. 25, 2099-2104. doi: 10.1002/mds.23234

Taylor, M. J. (2002). Non-spatial attentional effects on P1. Clin. Neurophysiol. 113, 1903-1908. doi: 10.1016/S1388-2457(02)00309-7

Thompson, E. (2015). Hamilton Rating Scale for Anxiety (HAM-A). Occup. Med. 65:601. doi: 10.1093/occmed/kqv054

Tomlinson, C. L., Stowe, R., Patel, S., Rick, C., Gray, R., and Clarke, C. E. (2010). Systematic review of levodopa dose equivalency reporting in Parkinson's disease. Mov. Disord. 25, 2649-2653. doi: 10.1002/mds.23429

Tsuboi, Y., Uchikado, H., and Dickson, D. W. (2007). Neuropathology of Parkinson's disease dementia and dementia with Lewy bodies with reference to striatal pathology. Parkinsonism Relat. Disord. 13(Suppl. 3), S221-S224. doi: 10.1016/S1353-8020(08)70005-1

Unsworth, N., and Engle, R. W. (2007). The nature of individual differences in working memory capacity: active maintenance in primary memory and controlled search from secondary memory. Psychol. Rev. 114, 104-132. doi: 10.1037/0033-295X.114.1.104

Vasavada, M. M., Wang, J., Eslinger, P. J., Gill, D. J., Sun, X., Karunanayaka, P., et al. (2015). Olfactory cortex degeneration in Alzheimer's disease and mild cognitive impairment. J. Alzheimers Dis. 45, 947-958. doi: 10.3233/JAD-1 41947

Vogel, E. K., and Luck, S. J. (2000). The visual N1 component as an index of a discrimination process. Psychophysiology 37, 190-203. doi: 10.1111/1469-8986. 3720190

Wang, G., Cheng, Q., Zeng, J., Bai, L., Liu, G. D., Zhang, Y., et al. (2008). Sleep disorders in Chinese patients with Parkinson's disease: validation study of a Chinese version of Parkinson's disease sleep scale. J. Neurol. Sci. 271, 153-157. doi: 10.1016/j.jns.2008.04.008

Wang, L., Kuroiwa, Y., Li, M., Wang, J., and Kamitani, T. (2001). Do P1 and N1 evoked by the ERP task reflect primary visual processing in Parkinson's disease? Doc. Ophthalmol. 102, 83-93.

Wenning, G. K., Shephard, B., Hawkes, C., Petruckevitch, A., Lees, A., and Quinn, N. (1995). Olfactory function in atypical parkinsonian syndromes. Acta Neurol. Scand. 91, 247-250. doi: 10.1111/j.1600-0404.1995.tb06998.x

Wright, M. J., Geffen, G. M., and Geffen, L. B. (1996). ERP measures of stimulus processing during an auditory oddball task in Parkinson's disease: evidence for an early information processing deficit. Parkinsonism Relat. Disord. 2, 13-21. doi: 10.1016/1353-8020(95)00024-0

Wyman-Chick, K. A., Martin, P. K., Barrett, M. J., Manning, C. A., and Sperling, S. A. (2017). Diagnostic accuracy and confidence in the clinical detection of cognitive impairment in early-stage parkinson disease. J. Geriatr. Psychiatry Neurol. 30, 178-183. doi: 10.1177/0891988717701001

Yamazaki, T., Kamijo, K., Kenmochi, A., Fukuzumi, S., Kiyuna, T., Takaki, Y., et al. (2000). Multiple equivalent current dipole source localization of visual eventrelated potentials during oddball paradigm with motor response. Brain Topogr. 12, 159-175. doi: 10.1023/A:1023467806268

Yu, J., Li, J., and Huang, X. (2012). The Beijing version of the Montreal Cognitive Assessment as a brief screening tool for mild cognitive impairment: a community-based study. BMC Psychiatry 12:156. doi: 10.1186/1471-244X-12-156

Conflict of Interest Statement: The authors declare that the research was conducted in the absence of any commercial or financial relationships that could be construed as a potential conflict of interest.

Copyright (C) 2019 Lin, Cui, Du, Li, He, Zhang, Fu, Huang, Gao, Li and Chen. This is an open-access article distributed under the terms of the Creative Commons Attribution License (CC BY). The use, distribution or reproduction in other forums is permitted, provided the original author(s) and the copyright owner(s) are credited and that the original publication in this journal is cited, in accordance with accepted academic practice. No use, distribution or reproduction is permitted which does not comply with these terms. 